\title{
Electrocorticogram encoding of upper extremity movement duration
}

\author{
Po T. Wang ${ }^{1}$, Christine E. King ${ }^{1}$, Colin M. McCrimmon ${ }^{1}$, Susan J. Shaw ${ }^{2,3}$, David E. Millett ${ }^{2,3}$, \\ Charles Y. Liu ${ }^{4,5}$, Luis A. Chui ${ }^{6}$, Zoran Nenadic ${ }^{1,7}$ and An H. Do
}

\begin{abstract}
Electrocorticogram (ECoG) is a promising longterm signal acquisition platform for brain-computer interface (BCI) systems such as upper extremity prostheses. Several studies have demonstrated decoding of arm and finger trajectories from ECoG high-gamma band $(80-160 \mathrm{~Hz})$ signals. In this study, we systematically vary the velocity of three elementary movement types (pincer grasp, elbow and shoulder flexion/extension) to test whether the high-gamma band encodes for the entirety of the movements, or merely the movement onset. To this end, linear regression models were created for the durations and amplitudes of high-gamma power bursts and velocity deflections. One subject with $8 \times 8$ high-density ECoG grid $(4 \mathrm{~mm}$ center-to-center electrode spacing) participated in the experiment. The results of the regression models indicated that the power burst durations varied directly with the movement durations (e.g. $R^{2}=0.71$ and slope $=1.0 \mathrm{~s} / \mathrm{s}$ for elbow). The persistence of power bursts for the duration of the movement suggests that the primary motor cortex (M1) is likely active for the entire duration of a movement, instead of providing a marker for the movement onset. On the other hand, the amplitudes were less co-varied. Furthermore, the electrodes of maximum $R^{2}$ conformed to somatotopic arrangement of the brain. Also, electrodes responsible for flexion and extension movements could be resolved on the high-density grid. In summary, these findings suggest that M1 may be directly responsible for activating the individual muscle motor units, and future BCI may be able to utilize them for better control of prostheses.
\end{abstract}

\section{INTRODUCTION}

Subdurally-recorded electrocorticogram (ECoG) is a promising long-term signal acquisition platform for braincomputer interface (BCI) systems, such as upper extremity prostheses. Several studies have shown that arm and finger trajectories [1], [2], [3], [4], [5], [6], [7] can be decoded from ECoG signals. However, the performance of these decoders has been modest. Thus, a better understanding of ECoG motor control may improve the design of trajectory decoders.

Our previous studies [8], [9] have demonstrated that the power of ECoG in the high- $\gamma$ band $(80-160 \mathrm{~Hz})$ strongly correlates with kinematic parameters of upper extremity move-

Work supported by the National Science Foundation (Award \#1134575)

${ }^{1}$ Department of Biomedical Engineering, University of California, Irvine (UCI), CA, USA \{ptwang, kingce, cmccrimm, znenadic\} @uci.edu

${ }^{2}$ Department of Neurology, Rancho Los Amigos National Rehabilitation Center (RLANRC), Downey, CA, USA \{millett, shaws\}@usc.edu

${ }^{3}$ Department of Neurology, University of Southern California (USC), Los Angeles, CA, USA

${ }^{4}$ Department of Neurosurgery, RLANRC, Downey, CA, USA cliu@usc.edu

${ }^{5}$ Department of Neurosurgery, USC, Los Angeles, CA, USA

${ }^{6}$ Department of Neurology, UCI, Irvine, CA, USA \{and, lchui\}@uci.edu

${ }^{7}$ Department of Electrical Engineering and Computer Science, UCI, Irvine, CA, USA ments. Specifically, the high- $\gamma$ power bursts have waveforms that resemble the velocity of elementary arm movements. However, these experiments did not systematically vary the movement velocity, and so it remains unclear whether a high- $\gamma$ power burst is responsible for the entire duration of movement or merely encodes for the movement onset. To this end, the velocity of various elementary upper extremity movements was systematically varied to better characterize motor control strategies in ECoG.

\section{METhODS}

\section{A. Signal Acquisition}

This study was approved by the Institutional Review Board of the Rancho Los Amigos National Rehabilitation Center. Subjects were recruited from a patient population undergoing temporary subdural electrode implantation for epilepsy surgery evaluation. Subject selection was limited to those with electrodes covering the primary motor cortex (M1) upper extremity representation area. Up to 64 channels of ECoG data were recorded using a pair of linked NeXus-32 bioamplifiers (Mind Media, Roermond-Herten, The Netherlands), and signals were acquired at a $2048-\mathrm{Hz}$ sample rate with common average referencing.

\section{B. Experimental Task}

A subset of elementary upper extremity movements [10] was performed on the side contralateral to the ECoG electrode implant: 1. pincer grasp and release (PG); 2. elbow flexion and extension (E); and 3. shoulder forward flexion and extension (SFE). Prior to each movement, an appropriate sensor to measure angular trajectory was mounted and calibrated using conventional goniometry. Specifically, the trajectory of PG was measured by a custom-made electrogoniometer [11], while E and SFE movements were measured by a gyroscope (Wii Motion Plus, Nintendo, Kyoto, Japan). The trajectory signals (position, $\theta$, and velocity, $\dot{\theta}$ ) were acquired using a microcontroller unit (Arduino, Smart Projects, Turin, Italy). ECoG data were synchronized with the trajectory signals using a common pulse train sent to both acquisition systems.

Each movement type was performed at fast, moderate, and slow speeds (see Table I for details) as guided by a video animation representing the moving joint. The video cued the subjects to fully flex the joint at the specified speed, followed by holding the joint stationary (idling) for a specific duration. This was then followed by a full extension movement and another idling period. The idling periods were introduced to prevent temporal overlapping of flexion and extension ECoG features, which we observed in earlier 
TABLE I

SUMMARY OF MOVEMENT TYPES AND SPEEDS. SUBJECTS WERE CUED TO PERFORM MAXIMUM FLEXION OR EXTENSION FOR THE SPECIFIED DURATION, AND IDLING WITHOUT RETURNING TO NEUTRAL POSITION.

\begin{tabular}{llll}
\hline Movement type & Fast & Moderate & Slow \\
\hline Pincer Grasp (PG) & $0.35 \mathrm{~s}$ & $0.75 \mathrm{~s}$ & $1.5 \mathrm{~s}$ \\
Elbow (E) & $0.65 \mathrm{~s}$ & $1.30 \mathrm{~s}$ & $2.6 \mathrm{~s}$ \\
Shoulder F/E (SFE) & $0.75 \mathrm{~s}$ & $1.5 \mathrm{~s}$ & $3.0 \mathrm{~s}$ \\
\hline Idle & $1.0 \mathrm{~s}$ & $2.0 \mathrm{~s}$ & $3.0 \mathrm{~s}$ \\
\hline
\end{tabular}

studies when these movements were executed in succession. The above cycle was repeated for a total of 40 times for each elementary movement type. In order to establish a signal baseline, subjects held the joint at a neutral position for $30 \mathrm{~s}$ before and after these video-guided cycles. Note that subjects familiarized themselves with the task by previewing the videos.

\section{Analysis}

As described in [9], the high $\gamma$-band power, $P_{\gamma}$, was obtained by calculating the instantaneous power envelope for each ECoG electrode:

$$
P_{\gamma}(t)=f\left(x_{\gamma}^{2}(t)\right)
$$

where $x_{\gamma}(t)$ is the bandpass filtered (80-160 Hz) ECoG signal, which is squared and then enveloped by a $1.5-\mathrm{Hz}$ low-pass filter, $f(\cdot)$.

The duration and amplitude of $P_{\gamma}$ bursts and $\dot{\theta}$ deflections during each flexion and extension movement were determined using criteria described in Fig. 1. Four separate linear regression models between $P_{\gamma}$ and $\dot{\theta}$ were generated for flexion durations, extension durations, flexion amplitudes, and extension amplitudes.

\section{RESULTS}

A 38-year-old male subject undergoing subdural electrode implantation for epilepsy surgery evaluation participated in this study. The subject had an $8 \times 8$ high-density ECoG electrode grid $(4 \mathrm{~cm} \times 4 \mathrm{~cm}, 4 \mathrm{~mm}$ center-to-center electrode spacing) placed over the right hemisphere covering the arm representation area of $\mathrm{M} 1$. While the subject completed all movement tasks, he had a tendency to decompose the slow PG movements into multiple small movements, and therefore this dataset was excluded from analysis. The ECoG grid location is reproduced in Fig. 2. Note that due to the presence of MRI incompatible metal inside his body, the ECoG grid could not be visualized using the typical MRICT co-registration procedure [12]. Instead, localization was performed by co-registering the central sulcus location using pre- and post-implantation CT scans.

The results of the regression models (summarized in Fig. 2 and Table II) indicated that the $P_{\gamma}$ burst duration varied directly with the movement duration for $\mathrm{E}$ and $\mathrm{SFE}$ movements. However, this relationship was weaker for the PG movement. Also, the amplitudes of $P_{\gamma}$ bursts and $\dot{\theta}$ deflections were less co-varied for all movement types. Representative scatter plots of these relationships are shown in Figs. 3 and 4.
TABLE II

THE MAXIMUM COEFFICIENT OF DETERMINATION $\left(\mathrm{R}^{2}\right)$, SLOPES, AND MEAN $P_{\gamma}$ VALUES FOR EACH LINEAR REGRESSION MODEL OVER THE M1 AREA. UNITS: $s$ FOR DURATIONS, $m a d$ FOR $P_{\gamma}$ AMPLITUdES, $\mathrm{mad} /(\% / \mathrm{s})$ FOR SLOPES OF AMPLITUDES. All $\mathrm{R}^{2}$ VAlues ARE STATISTICALLY SIGNIFICANT INCLUDING $\mathrm{R}^{2}=0.10(\mathrm{P}=0.0075)$.

\begin{tabular}{llllll}
\hline \multicolumn{2}{l}{ Movement } & \multicolumn{2}{c}{ Duration } & \multicolumn{2}{c}{ Amplitude } \\
& & Flexion & Extension & Flexion & Extension \\
\hline PG & $\mathrm{R}^{2}$ & 0.42 & 0.34 & 0.10 & 0.23 \\
& Electrode & $\mathrm{G} 37$ & $\mathrm{G} 30$ & $\mathrm{G} 23$ & $\mathrm{G} 9$ \\
& Slope & 0.92 & 0.61 & -0.0094 & -0.012 \\
& $P_{\gamma}$ Value & 0.74 & 0.74 & 3.68 & 2.63 \\
\hline $\mathrm{E}$ & $\mathrm{R}^{2}$ & 0.71 & 0.62 & 0.41 & 0.55 \\
& Electrode & $\mathrm{G} 37$ & $\mathrm{G} 37$ & $\mathrm{G} 36$ & $\mathrm{G} 19$ \\
& Slope & 1.0 & 0.85 & 0.081 & -0.080 \\
& $P_{\gamma}$ Value & 1.50 & 1.55 & 22.58 & 15.69 \\
\hline SFE & $\mathrm{R}^{2}$ & 0.69 & 0.71 & 0.30 & 0.33 \\
& Electrode & $\mathrm{G} 27$ & $\mathrm{G} 20$ & $\mathrm{G} 29$ & $\mathrm{G} 26$ \\
& Slope & 1.0 & 1.2 & -0.085 & -0.069 \\
& $P_{\gamma}$ Value & 1.63 & 1.62 & 13.99 & 18.26 \\
\hline
\end{tabular}

\section{Discussion}

The persistence of $P_{\gamma}$ bursts for the duration of the movement suggests that M1 is likely active for the entire duration of a movement instead of providing a marker for the movement onset. For each individual flexion and extension in the E and SFE movements, the duration of the $P_{\gamma}$ burst was approximately equal to the duration of the $\dot{\theta}$ deflection (as evidenced by slope of $\sim 1$ ). This relationship was true for a range of velocities (see Table II), including the slow speed movements. This raises the possibility that M1 neurons directly activate motor units responsible for the movements. Furthermore, the slope of the duration regression line was slightly higher for SFE. This suggests that for larger joints, the $P_{\gamma}$ activity may encode for acceleration or a combination of both velocity and acceleration, whose deflection lasts longer than that of $\dot{\theta}$.

On the other hand, even though the $\mathrm{R}^{2}$ values corresponding to the amplitudes of $P_{\gamma}$ bursts and $\dot{\theta}$ deflections were statistically significant $(\mathrm{p}=0.0075)$, the majority of variance was unaccounted for. Only the $\mathrm{R}^{2}$ and slopes for $\mathrm{E}$ movement supported the original hypothesis $\left(P_{\gamma}\right.$ burst amplitude correlates with the movement deflection amplitude). The possibility of other variables (e.g. torque, muscle activity) being encoded by this feature may explain the remaining variance. The inherent variability of the peak values of the $P_{\gamma}$ bursts may be another contributing factor.

The spatial distribution (Fig. 2) of the maximum $\mathrm{R}^{2}$ across movement types conformed to a somatotopic arrangement in M1, in which proximal to distal movements were represented in a medial to lateral manner. In addition, the area of high $\mathrm{R}^{2}$ increased for $\mathrm{E}$ and $\mathrm{SFE}$ movements, compared to $\mathrm{PG}$ movements. This can be explained by the stronger $P_{\gamma}$ bursts (higher $P_{\gamma}$ amplitudes, see Table II), leading to a larger spread of movement-modulated high- $\gamma$ ECoG activity by volume conduction. This in turn allowed high $\mathrm{R}^{2}$ values to be established over wider brain areas.

The locations of maximum $\mathrm{R}^{2}$ differed between flexion 


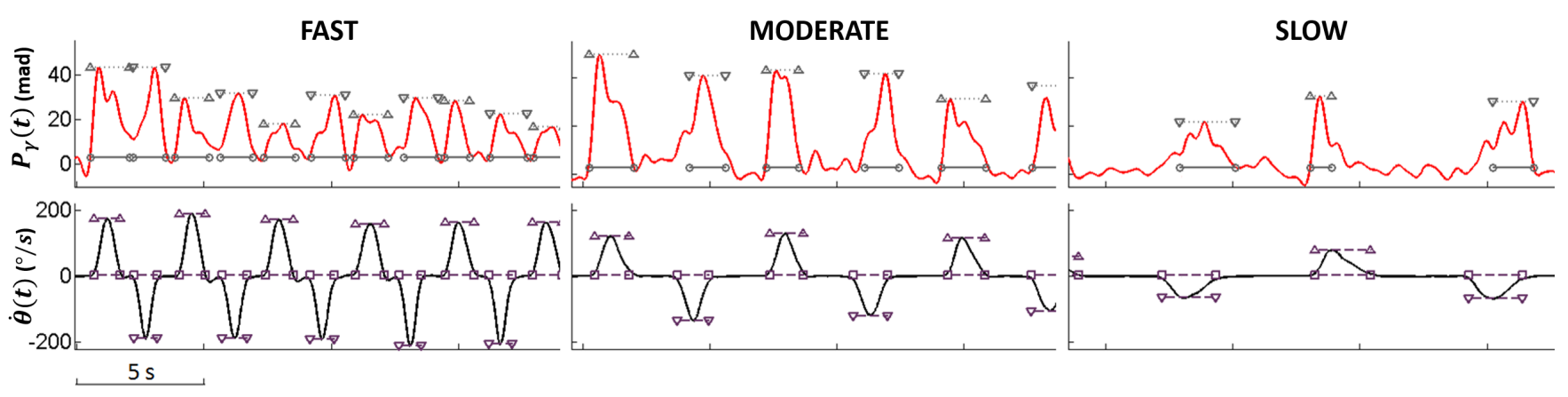

Fig. 1. Representative time series of the three different speeds for SFE movements. The top traces are $P_{\gamma}$ at electrode G37, and the bottom traces are $\dot{\theta}$. The $P_{\gamma}$ signal from each electrode was standardized such that the median $=0$ and the median absolute deviation $(\mathrm{mad})=1 \mathrm{during}$ the initial 30 -s idling period (not shown). The widths (durations) of both the $P_{\gamma}$ bursts and $\dot{\theta}$ deflections were determined at the 3-mad threshold (circle and square segments) or between local minima. The amplitudes were determined by averaging over 5 points around the extremum within each segment and are indicated by triangles.
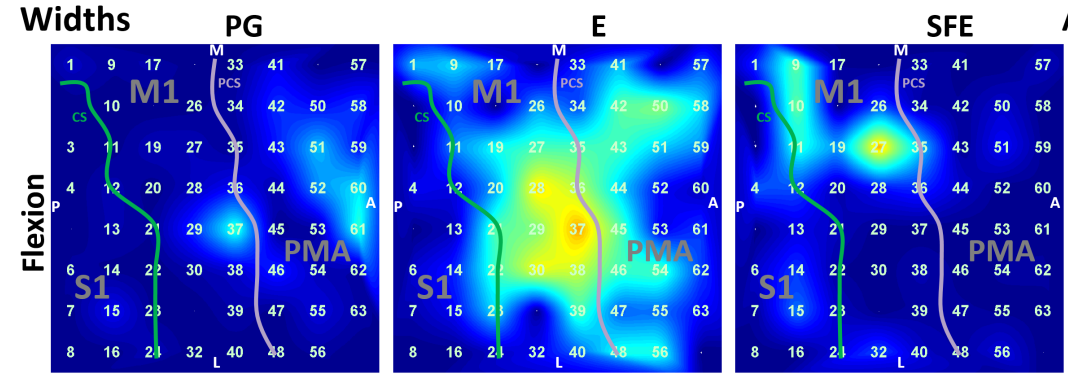

Amplitudes E
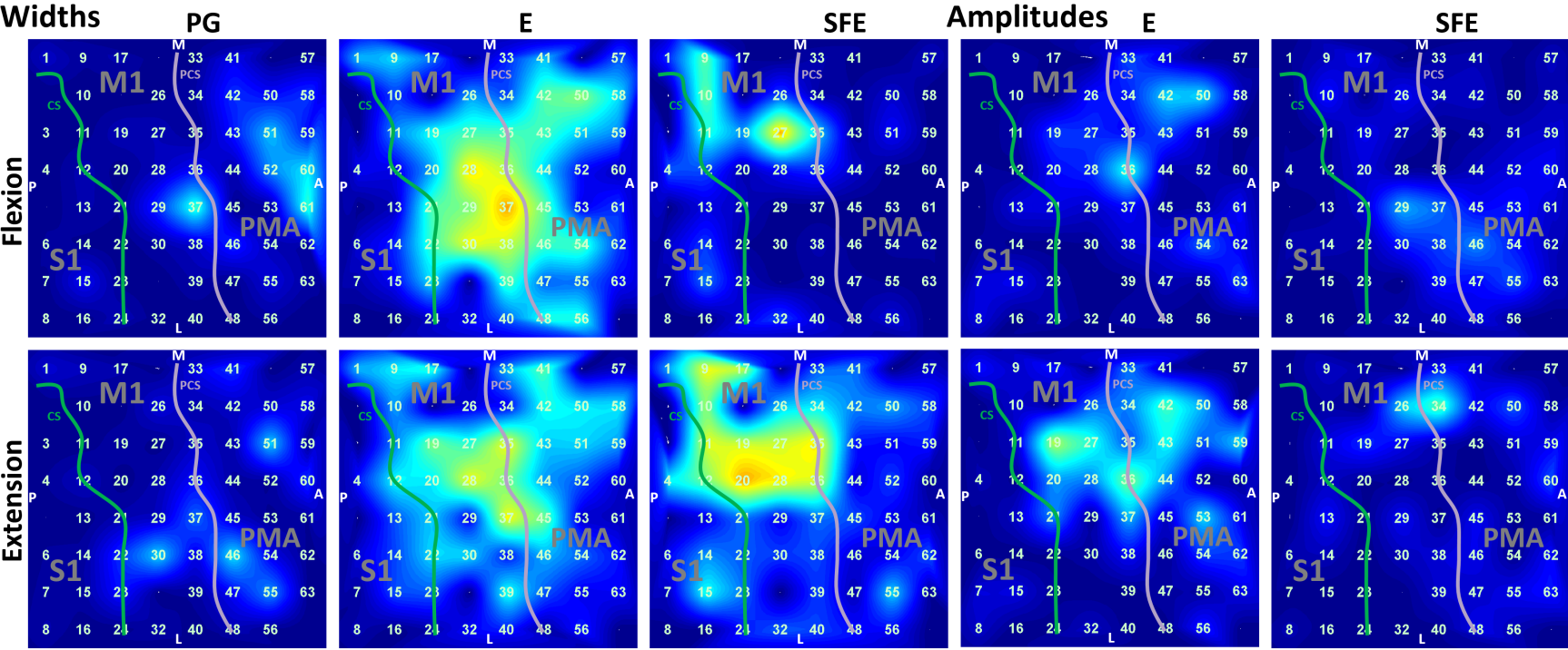

Fig. 2. Spatial distribution of $\mathrm{R}^{2}$ values between the durations of the $P_{\gamma}$ bursts and $\dot{\theta}$ deflections, and between the amplitudes of the $P_{\gamma}$ bursts and $\dot{\theta}$ deflections. Note that amplitudes during $\mathrm{PG}$ are not shown since the $\mathrm{R}^{2}$ values are all $<0.23$. Abbr: $\mathrm{A}=\mathrm{Anterior}, \mathrm{P}=\mathrm{Posterior}, \mathrm{M}=\mathrm{Medial}, \mathrm{L}=\mathrm{Lateral}$, M1 = Primary motor cortex, S1 = Primary sensory cortex, PMA = Pre-motor area, CS $=$ Central sulcus, PCS $=$ Pre-central sulcus.

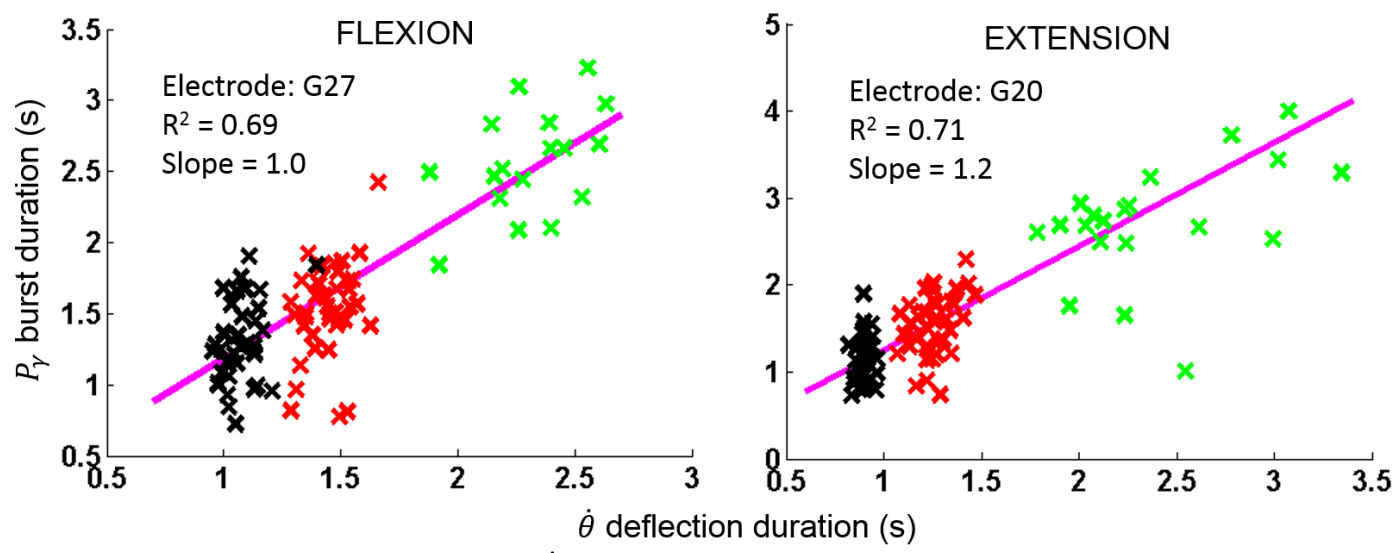

Fig. 3. Relationship between the durations of $P_{\gamma}$ bursts and $\dot{\theta}$ deflections for SFE movement. Black crosses (fast speed), red crosses (moderate speed), green crosses (slow speed). A best-fit line is also shown for each regression model. 


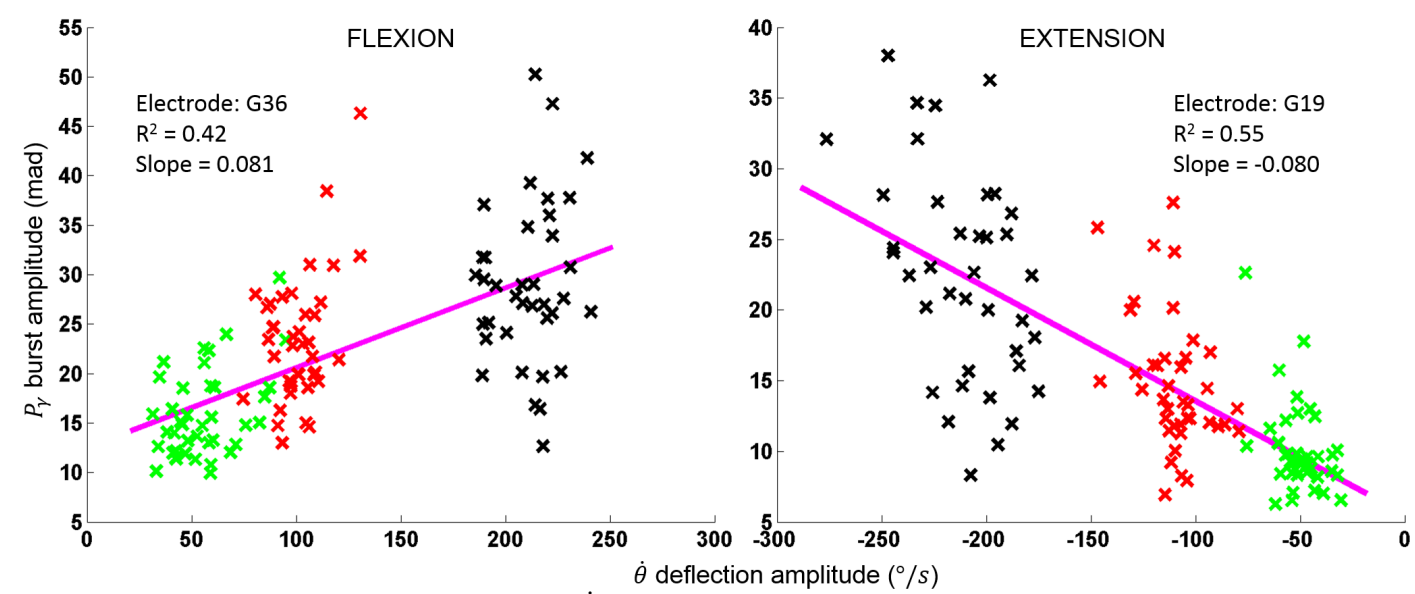

Fig. 4. Relationship between the amplitudes of $P_{\gamma}$ bursts and $\dot{\theta}$ deflections for E movement. Black crosses (fast speed), red crosses (moderate speed), green crosses (slow speed). A best-fit line is also shown for each regression model.

and extension for each movement type, suggesting that there exist separate neuronal generators responsible for each movement direction. These generators appeared to be sufficiently resolved in space using a high-density ECoG grid, which makes it a promising signal acquisition platform for future BCI applications. In summary, the strong $\mathrm{R}^{2}$ for durations, the presence of their somatotopic arrangement, and the separation of flexion and extension generators further reinforce the possibility that M1 cortical neurons are directly responsible for activating muscle motor units.

Finally, it can be observed that $P_{\gamma}$ bursts did not fully return to the noise threshold between flexion and extension movements at fast speed (see Fig. 1). Adjusting future experiments to increase the idling period to $2-3 \mathrm{~s}$ may better isolate the ECoG signals encoding for these movements and may improve the $\mathrm{R}^{2}$ value.

\section{CONCLUSION}

This study explored the characteristics of high- $\gamma$ power bursts at varying movement velocities. The results support the hypothesis that M1 is active for the entire duration of a movement instead of marking the onset of movement. They also suggest that M1 may be directly responsible for activating the individual muscle motor units. Our future work will focus on corroborating these findings and testing this hypothesis in a larger cohort of subjects. Ultimately, this may improve our understanding of physiological processes underlying upper extremity movement, which in conjunction with high-density ECoG grids, may lead to the design of better BCI systems for upper extremity prosthesis control.

\section{ACKNOWLEDGMENTS}

We thank Angelica Nguyen for her assistance with the experiments and Andrew Schombs for building the velocity acquisition system.

\section{REFERENCES}

[1] P. T. Wang, E. J. Puttock, C. E. King, A. Schombs, J. J. Lin, M. Sazgar, F. P. K. Hsu, S. J. Shaw, D. E. Millett, C. Y. Liu, L. A. Chui, A. H. Do, and Z. Nenadic, "State and trajectory decoding of upper extremity movements from electrocorticogram," in Proc 6th Int IEEE EMBS Conf Neural Eng, pp. 969-972, 2013.
[2] W. Wang, J. L. Collinger, A. D. Degenhart, E. C. Tyler-Kabara, A. B. Schwartz, D. W. Moran, R. K. Vinjamuri, R. C. Ashmore, D. J. Weber, B. Wodlinger, J. W. Kelly, and M. L. Boninger, "An electrocorticographic brain interface in an individual with tetraplegia," PLOS ONE, vol. 8, p. e55344, 2013.

[3] T. Yanagisawa, M. Hirata, Y. Saitoh, H. Kishima, K. Matsushita, T. Goto, R. Fukuma, H. Yokoi, Y. Kamitani, and T. Yoshimine, "Electrocorticographic control of a prosthetic arm in paralyzed patients," Ann Neurol, vol. 71, no. 3, pp. 353-361, 2012.

[4] K. J. Miller, S. Zanos, E. E. Fetz, M. den Nijs, and J. G. Ojemann, "Decoupling the cortical power spectrum reveals real-time representation of individual finger movements in humans.," J Neurosci, vol. 29 no. 10, pp. 3132-3137, 2009.

[5] S. Acharya, M. S. Fifer, H. L. Benz, N. E. Crone, and N. V. Thakor, "Electrocorticographic amplitude predicts finger positions during slow grasping motions of the hand," J Neural Eng, vol. 7, no. 4, p. 046002 , 2010

[6] J. Kubánek, K. J. Miller, J. G. Ojemann, J. R. Wolpaw, and G. Schalk, "Decoding flexion of individual fingers using electrocorticographic signals in humans," J Neural Eng, vol. 6, no. 6, p. 66001, 2009.

[7] J. C. Sanchez, A. Gunduz, P. R. Carney, and J. C. Principe, "Extraction and localization of mesoscopic motor control signals for human ECoG neuroprosthetics.," J Neurosci Meth, vol. 167, no. 1, pp. 63-81, 2008.

[8] P. T. Wang, C. E. King, A. Schombs, J. J. Lin, M. Sazgar, F. P. K. Hsu, S. J. Shaw, D. E. Millett, C. Y. Liu, L. A. Chui, Z. Nenadic, and A. H. Do, "Electrocorticographic gamma band power encodes the velocity of upper extremity movements," in Proc 5th Int BrainComputer Interface Meeting, Article ID 120, 2013.

[9] P. T. Wang, C. E. King, A. Schombs, J. J. Lin, M. Sazgar, F. P. K. Hsu, S. J. Shaw, D. Millett, C. Y. Liu, L. A. Chui, Z. Nenadic, and A. H. Do, "Electrocorticogram encoding of upper extremity movement trajectories," in Proc 6th Int IEEE EMBS Conf Neural Eng, pp. 1429_ 1432, 2013.

[10] A. H. Do, P. T. Wang, C. E. King, A. Schombs, J. J. Lin, M. Sazgar, F. P. K. Hsu, S. J. Shaw, D. E. Millett, C. Y. Liu, A. A. Szymanska, L. A. Chui, and Z. Nenadic, "Sensitivity and specificity of upper extremity movements decoded from electrocorticogram," in Proc 35th Ann Int Conf IEEE EMBS, pp. 5618-5621, 2013.

[11] P. T. Wang, C. E. King, A. H. Do, and Z. Nenadic, "A durable, lowcost electrogoniometer for dynamic measurement of joint trajectories," Med Eng Phys, vol. 33, no. 5, pp. 546-552, 2011.

[12] P. T. Wang, C. E. King, S. J. Shaw, D. E. Millett, C. Y. Liu, L. A. Chui, Z. Nenadic, and A. H. Do, "A co-registration approach for electrocorticogram electrode localization using post-implantation MRI and CT of the head," in Proc 6th Int IEEE EMBS Conf Neural Eng, pp. 525-528, 2013. 